\title{
Un Método de Optimización Proximal para Problemas de Localización Cuasi-convexa
}

\author{
Miguel A. Cano Lengua ${ }^{1}$, Erik A. Papa Quiroz ${ }^{2}$ \\ ${ }^{1}$ Facultad de Ciencias Naturales y Matemática -FCNM/ Universidad Nacional del Callao \\ Callao- Perú \\ ${ }^{2}$ Facultad de Ciencias Naturales y Matemática -FCNM/ Universidad Nacional del Callao \\ Callao- Perú
}

\section{RESUMEN}

El problema de localización es de gran interés para poder establecer de manera óptima diferentes demandas de ubicación en el sector estatal o privado. El modelo de este problema se reduce generalmente a un problema de optimización matemática.

En el presente trabajo presentamos un método de optimización proximal para resolver problemas de localización donde la función objetivo es cuasi-convexa y no diferenciable. Probamos que las iteraciones dadas por el método están bien definidas y bajo algunas hipótesis sobre la función objetivo probamos la convergencia del método.

Descriptores: Método del punto proximal, teoría de localización, convergencia global, función cuasi-convexa

\section{ABSTRACT}

The localization problem is of great interest to establish the optimal location of the different demands in the state or private sector. The model of this problem is generally reduced to solve a mathematical optimization problem.

In the present work we present a proximal optimization method to solve localization problems where the objective function is non differentiable and quasiconvex. We prove that the iterations of the method are well defined and under some assumption on the objective function we prove the convergence of the method.

\section{Keywords:}

Proximal point method, localization theory, global convergence, quasiconvex function

\section{Introducción}

En el campo de la matemática aplicada, existen diversas áreas que dan solución a problemas vinculados a las ciencias e ingenierías, una de ellas es la Optimización Matemática que estudia la forma de encontrar la mejor solución a un problema dado dentro de todas las posibles alternativas.

El modelo general de optimización está dado por:

$$
\begin{aligned}
& \text { Opt.f(x) } \\
& \text { s.a: } \\
& g_{i}(x) \leq 0 ; \forall i=1, \ldots, m, \\
& h_{j}(x)=0 ; \forall j=1, \ldots, p,
\end{aligned}
$$

donde $f: R^{n} \rightarrow R$ es una función dada, Opt.f(x) significa minimizar o maximizar la función $f$, además $g_{i}: R^{n} \rightarrow R \quad$ y $\quad h_{j}: R^{n} \rightarrow R$ son funciones dadas.

Una clase particular y bien amplia del modelo $(P)$ es la optimización cuasi-convexa (problemas donde la función objetivo $f$ es cuasi-convexa, esto es, $f(\lambda x+(1-\lambda) y) \leq \max \{f(x), f(y)\}, \forall \lambda \in[0,1], \forall x, y \in R^{n}$, $\mathrm{y}$ las funciones que definen las restricciones $g_{i} \mathrm{y}$ $h_{j}$ son cuasi-convexas). 
Este problema fue estudiado en el año 1951 por Arrow y Enthoven [1], motivados por las aplicaciones de las preferencias y utilidades en la teoría del consumidor y posteriormente por diversos investigadores como Kannai, [5].

Por otro lado, problemas de localización tienen una gran importancia en las ciencias e ingenierías. Estos entraron en las ciencias inicialmente como problemas matemáticos donde personajes ilustres como Fermat, Torriceli, Silvester, o Steiner propusieron ingeniosos métodos para resolverlos.

En el siglo XX los problemas de localización se extendieron a varias disciplinas y actualmente tienen una gran variedad de aplicaciones en diversas áreas de investigación.

Este trabajo está destinado a resolver problemas de localización modelados de la siguiente forma general

$$
(P I)\left\{\begin{array}{c}
\min \\
s . a: \\
x \in R^{n}
\end{array}\right.
$$

donde $f: R^{n} \rightarrow R$ es una función real no diferenciable y cuasi-convexa (ver Sección 2 para corroborar el modelo ).

Para resolver el problema planteado extenderemos el método del punto proximal, ver [6] y[7], que para el caso donde la función $f$ es convexa, genera una sucesión de puntos, a partir de un punto dado $x^{\circ} \in R$, tal que: Dado $k=1,2,3, \ldots$,

Si $0 \in \partial_{c} f\left(x^{k-1}\right)$ entonces finaliza (donde $\partial_{c} f$ es el subdiferencial convexo).

Caso contrario, se busca un $x^{k} \in R^{n}$ tal que:

$x^{k}=\arg \min \left\{f(x)+\frac{\lambda_{k}}{2}\left\|x-x^{k-1}\right\|^{2}: x \in R^{n}\right\}$

Fué demostrado, ver [4], que si $f$ es convexa, propia y que la sucesión $\left\{\lambda_{k}\right\}$ satisface:

$$
\sum_{k=1}^{+\infty} \frac{1}{\lambda_{k}}=+\infty
$$

entonces la sucesión $\left\{f\left(x^{k}\right)\right\}$ converge al ínfimo de t y si además el conjunto de soluciones óptimas no es vacío, entonces la sucesión converge para una solución del problema.
Para el caso cuando $f$ es cuasi-convexa (y por lo tanto para el problema de localización que deseamos resolver), debemos observar que debido a la no convexidad de $t$, el problema (1) no es necesariamente un problema convexo, más aún, no es necesariamente cuasi-convexo. Es por eso, que la iteración (1) puede ser más difícil de resolver que el problema $(P I)$.

Para superar esta dificultad, proponemos una extensión del método del punto proximal usando la iteración:

$$
0 \in \hat{\partial}\left(f(.)+\left(\frac{\lambda_{k}}{2}\right)\left\|\cdot-x^{k-1}\right\|^{2}\right)\left(x^{k}\right)
$$

donde $\hat{\partial}$ es el subdiferencial de Clarke,

ver Sección 3 para una definición rigurosa de este subdiferencial.

Esta iteración es más razonable de resolver desde el punto de vista teórico y práctico ya que en vez de encontrar el punto $x^{k}$ que minimice la función $f()+.\left(\frac{\lambda_{k}}{2}\right)\left\|\cdot-x^{k-1}\right\|^{2}$, sólo necesitamos encontrar un punto crítico del mismo. Así reducimos el costo computacional en cada iteración con respecto al método proximal clásico.

En este trabajo probamos la convergencia del método proximal usando la iteración (2) bajo la hipótesis que la función $f$ es localmente Lipschitz , cuasi-convexa y no necesariamente diferenciable.

La organización de este trabajo es la siguiente:

En la Sección 1, presentamos las herramientas necesarias para el desarrollo de este trabajo, entre otros veremos algunos resultados del análisis convexo, subdiferenciabilidad para funciones convexas. En la Sección 2 se presenta el modelo de localización cuasi-convexo. En la Sección 3 presentamos la teoría del subdiferencial de Clarke (ver referencias [2] y [3] para mayor profundidad). En la sección 4, parte central del trabajo, presentamos el método propuesto y sus resultados de convergencia. En la sección 5, presentamos la implementación del método para algunas funciones y finalmente damos la bibliografía como fuente de información. 


\subsection{Definiciones Básicas}

Definición 1.2.1 Una función $f: R^{n} \rightarrow R \cup\{+\infty\}$ es llamada convexa en $R^{n}$ si para todo $x, y \in R^{n}$ y para todo $\lambda \in[0,1]$ se cumple que:

$$
f(\lambda x+(1-\lambda) y) \leq \lambda f(x)+(1-\lambda) f(y) .
$$

Definición 1.2.2 Una función $f: R^{n} \rightarrow R \cup\{+\infty\}$ es llamada cuasi-convexa en $R^{n}$ si para todo $x, y \in R^{n}$ y para todo $\lambda \in[0,1]$ se cumple que:

$$
f(\lambda x+(1-\lambda) y) \leq \max \{f(x), f(y)\} .
$$

Definición 1.2.3 Sea $f: R^{n} \rightarrow R \cup\{+\infty\}$ una función. Decimos que $\bar{x} \in R^{n}$ es un mínimo local de $f$ si existe $\varepsilon>0$ tal que $f(\bar{x}) \leq f(x), \forall x \in B(\bar{x}, \varepsilon)$.

Decimos que $\bar{x} \in R^{n}$ es un mínimo global de $f$ si $f(\bar{x}) \leq f(x), \forall x \in R^{n}$.

Definición 1.2.4 Una función $f: R^{n} \rightarrow R \cup\{+\infty\}$ es localmente lipschitziana con constante $k>0$ en $x \in R^{n}$ si existe algún $\varepsilon \geq 0$ tal que;

$$
|f(y)-f(z)| \leq k\|y-z\| \text { para todo } z, y \in B(x, \varepsilon) \text {. }
$$

Definición 1.2.5 Una función $f: R^{n} \rightarrow R \cup\{+\infty\}$ es semicontinua inferior en $\bar{x} \in R^{n}$ si para toda sucesión $\left\{x^{l}\right\}$ convergente a $\bar{x}$, se tiene que:

$$
f(\bar{x}) \leq \liminf _{l \rightarrow \infty} f\left(x^{l}\right) .
$$

Definición 1.2.6 Sea $f: R^{n} \rightarrow R \cup\{+\infty\}$. Esta function es llamada función propia si:

(a) $\operatorname{domf} \neq \phi$.

(b) $\forall x \in$ domf , tenemos que $f(x)>-\infty$.

\subsection{Subdiferenciabilidad}

Definición 1.3.1 Se define la derivada direccional de $f$ en $x$ en la dirección $v \in R^{n}$ como:

$$
f^{\prime}(x, v)=\lim _{t \downarrow 0} \frac{f(x+t v)-f(x)}{t} .
$$

Si $f$ es diferenciable en $x$, entonces la derivada direccional existe en toda dirección $v \in R^{n}, f^{\prime}(x, v)$ es una función lineal en $v$ y tenemos la siguiente relación:

$$
f^{\prime}(x, v)=\langle\nabla f(x) ; v\rangle .
$$

Definición 1.3.2 Sea $f: R^{n} \rightarrow R \cup\{+\infty\}$ una función convexa. Dado $x \in R^{n}, \quad s \in R^{n}$ es un subgradiente de $f$ en $x$ si:

$$
f(y) \geq f(x)+\langle s ; y-x\rangle, \forall y \in R^{n} .
$$

El conjunto de todos los subgradientes de $f$ en $x$ es llamado subdiferencial convexo de $f$ en $x$ y es denotado por $\partial f_{c}(x)$, esto es:

$\partial f_{c}(x)=\left\{s \in R^{n}: f(y) \geq f(x)+\langle s ; y-x\rangle, \forall y \in R^{n}\right\}$.

Proposición 1.3.1 Sea $f: R^{n} \rightarrow R \cup\{+\infty\}$ una función convexa y $\bar{x} \in \operatorname{int}(\operatorname{domf})$. Si $f$ es diferenciable en $\bar{x}$, entonces : $\partial f_{c}(\bar{x})=\{\nabla f(\bar{x})\}$.

Ejemplo 1.3.1: Sea la función $f(x)=|x|$ (Figura 1) es fácil de verificar que el subdiferencial convexo de $f$ en $x$ es:

$$
\partial f_{c}(x)=\left\{\begin{array}{c}
1 ; x>0 \\
-1 ; x<0 \\
{[-1,1] ; x=0}
\end{array}\right.
$$

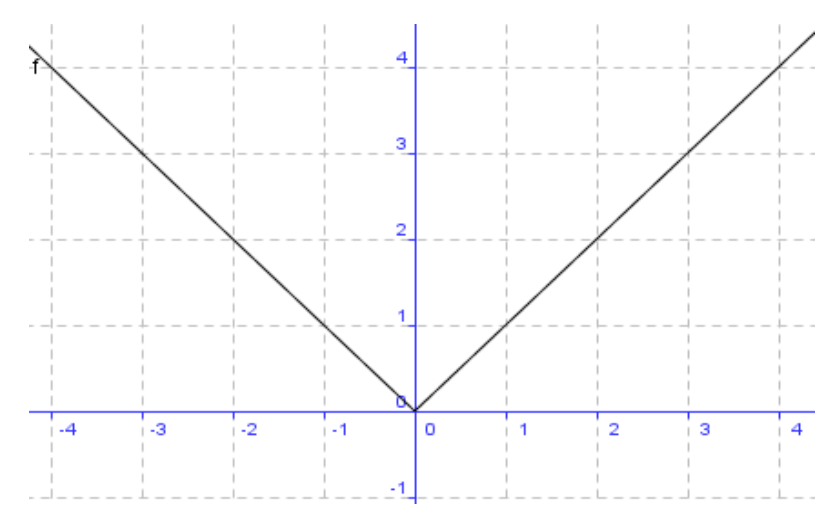

Figura 1 del ejemplo $f(x)=|x|$

Ejemplo 1.3.2: Sea la función definida por:

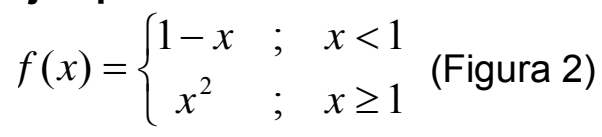


es fácil de verificar que el subdiferencial convexo de $t$ en $x$ es:

$$
\partial f_{c}(x)=\left\{\begin{array}{rr}
1 & ; x<1 \\
2 x & ; x>1 \\
{[-1,2]} & ; x=1
\end{array}\right.
$$

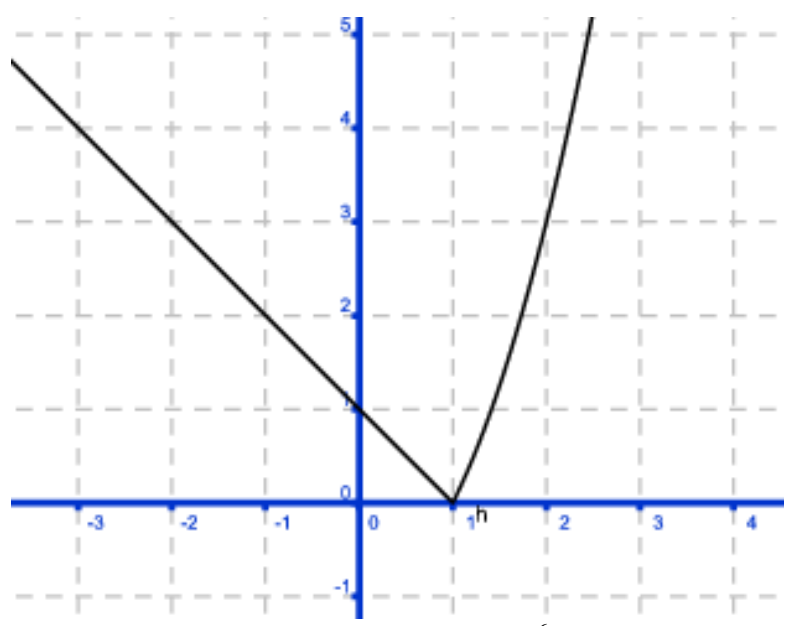

Figura 2 del ejemplo $f(x)=\left\{\begin{array}{cc}1-x & ; x<1 \\ x^{2} & ; \quad x \geq 1\end{array}\right.$

\section{Modelo de Localización Cuasi-convexo}

Un ejemplo de problema de localización es el de ubicar una central de bomberos el cual pueda dar servicio en el menor tiempo posible a determinados puntos como por ejemplo a una Iglesia, colegio, hospital, universidad, comisaria, centro recreacional, banco, municipalidad, centro de convenciones y un condominio, ver (Figura 3).

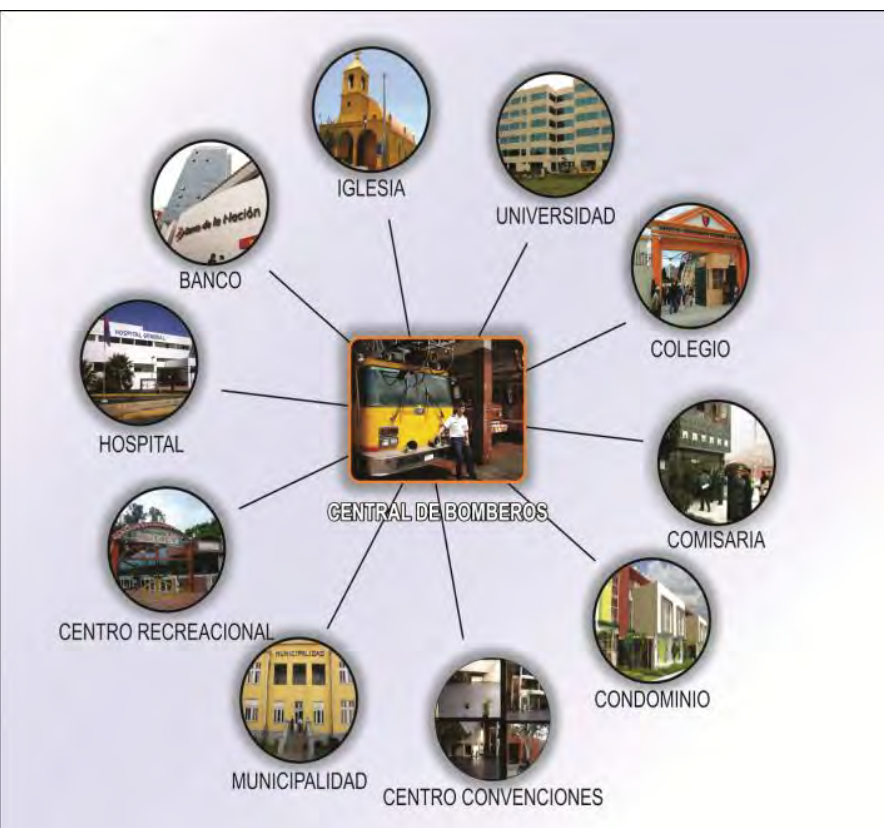

Figura 3 Central de bomberos
A seguir presentamos un modelo formal de localización cuasi-convexo.

Sea $D=\left\{d_{1}, d_{2}, \ldots, d_{p}\right\} \subset R^{n}, n \geq 2$, un conjunto de $p$ puntos de diferentes demandas y sea $x \in R^{n}$ la ubicación de una instalación a ser elegida.

Si $C_{i}, i=1, \ldots, p$, son conjuntos compactos con $0 \in \operatorname{int}\left(C_{i}\right), \quad\left(\operatorname{int}\left(C_{i}\right) \quad\right.$ denota el interior de $\left.C_{i}\right)$ definimos la distancia entre $x$ y $d_{i}$ por $\gamma_{C_{i}}\left(x-d_{i}\right)$ donde $\gamma_{C_{i}}$, es el funcional de Minkowsky del conjunto $C_{i}$, es decir, $\quad \gamma_{C_{i}}(x)=\inf \left\{t>0: x \in t C_{i}\right\}$ observe que si $C_{i}$ es la bola unitaria sobre $R^{n}$ entonces $\gamma_{C_{i}}(x)$ es la distancia euclideana de $x$ a 0 . Para introducir el modelo, definimos la función $\gamma: R^{n} \rightarrow R_{+}^{p}$ tal que

$$
\gamma(x)=\left(\gamma_{C_{1}}\left(x-d_{1}\right), \ldots, \gamma_{C_{p}}\left(x-d_{p}\right)\right),
$$

y suponemos que $f_{i}: R^{p} \rightarrow R, i=1, \ldots, p$, es una función no decreciente sobre $R_{+}^{p}$, esto es, si $x, y \in R_{+}^{p}$ satisface $\quad x_{i} \leq y_{i}, \forall i=1, \ldots, p, \quad$ entonces $f_{i}(x) \leq f_{i}(y)$.

El modelo es dado por:

donde

$$
\min \left\{\phi(x): x \in R^{n}\right\}
$$

$$
\phi(x)=\max _{1 \leq x \leq p}\left\{\phi_{i}(x)\right\}
$$

con $\phi_{i}: R^{n} \rightarrow R$ definido por $\phi_{i}(x)=f_{i}(\gamma(x))$, para cada $i=1, \ldots, p$

Si la función $f_{i}: R^{p} \rightarrow R$ es cuasi-convexa sobre $R_{+}^{p}$ entonces es posible probar que la función $\phi$ es cuasi-convexa sobre $R^{n}$.

\section{Subdiferencial de Clarke}

\section{Definición $\quad 3.1 \quad$ Dado $f: R^{n} \rightarrow R \cup\{+\infty\}$}

localmente lipschitziana en el punto $x \in R^{n}$. La derivada direccional generalizada de $f$ en $x$, en la dirección de $v \in R^{n}$, es definida por:

$$
f^{0}(x, v)=\lim _{\substack{y \rightarrow x \\ t \downarrow 0}} \operatorname{Sup} \frac{f(y+t v)-f(y)}{t}
$$


Debemos notar que $f^{0}$ siempre existe gracias a la condición de localmente lipschitziana de la función $t$.

Definición $\quad 3.2$ Sea $f: R^{n} \rightarrow R \cup\{+\infty\}$ una función localmente lipschitziana en el punto $x \in R^{n}$, entonces el subdiferencial, en el sentido de Clarke, de $f$ en $x$ es el conjunto

$$
\hat{\partial} f(x)=\left\{\xi \in R^{n}: f^{0}(x, v) \geq\langle\xi ; v\rangle, \forall v \in R^{n}\right\} .
$$

Cada elemento de $\xi \in \hat{\partial} f(x)$, es llamado subgradiente de $f$ en $x$, en el sentido de Clarke.

Definición 3.3 Sea $f: R^{n} \rightarrow R \cup\{+\infty\}$ una función propia, el conjunto de subgradientes generalizados (también llamado límite subdiferencial) de $f$ en $x \in R^{n}$, denotado por $\partial f(x)$ es definido como:

$$
\partial f(x)=\left\{s \in R^{n}: \exists x^{l} \rightarrow x, f\left(x^{l}\right) \rightarrow f(x), \exists s^{l} \in \hat{\partial} f\left(x^{l}\right) \rightarrow s\right\} .
$$

Ejemplo 3.1: Sea la función $\quad g(x)=x^{2} \operatorname{sen}\left(\frac{1}{x}\right)$

(Figura 4) es fácil de verificar que el subdiferencial Clarke de $f$ en 0 es:

$$
\hat{\partial} f(0)=[-1,1]
$$

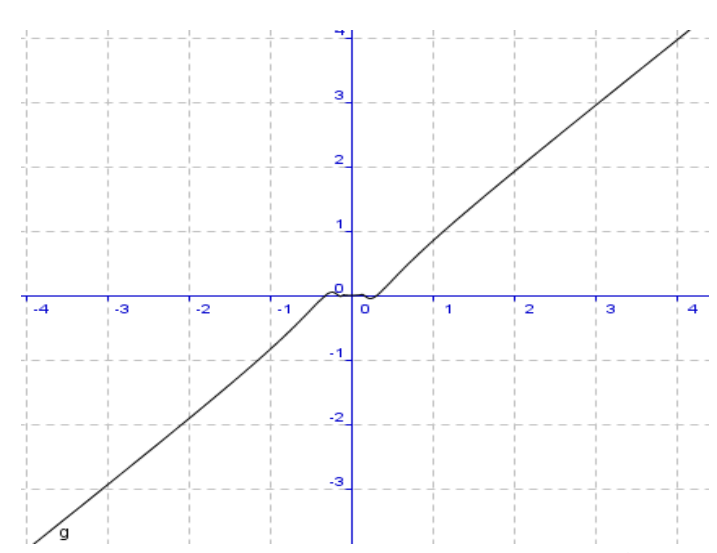

Figura 4 del ejemplo $g(x)=x^{2} \operatorname{sen}\left(\frac{1}{x}\right)$

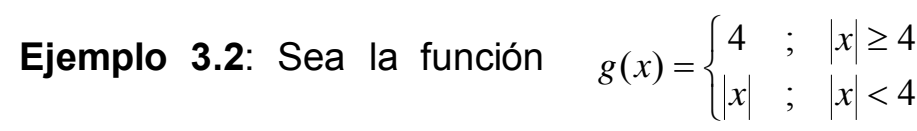
(Figura 5) es fácil de verificar que el subdiferencial de Clarke está dado por:

$$
\hat{\partial} g(x)=\left\{\begin{array}{ccc}
0 & ; & x<-4 \\
{[-1,0]} & ; & x=-4 \\
-1 & ; & -4<x<0 \\
{[-1,1]} & ; & x=0 \\
1 & ; & 0<x<4 \\
{[0,1]} & ; & x=4 \\
0 & ; & x>4
\end{array}\right.
$$

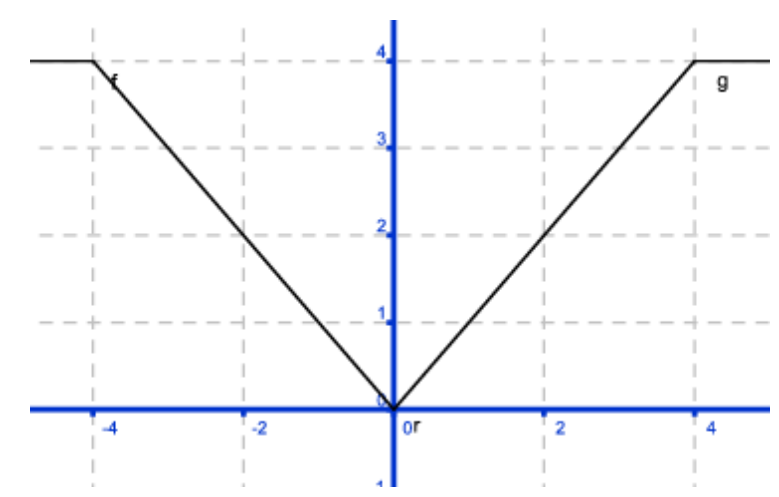

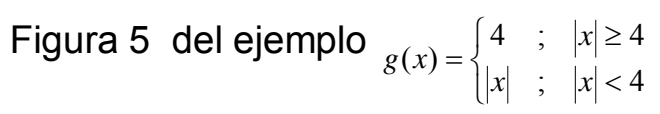

\section{Método del Punto Proximal}

Consideremos el problema:

$$
(P)\left\{\begin{array}{c}
\min \\
s . a: \\
x \in R^{n}
\end{array}\right.
$$

donde $f: R^{n} \rightarrow R \cup\{+\infty\}$ es una función propia, semicontinua inferior y $R^{n}$ es el espacio euclidiano con norma $\|$.$\| . El método del Punto Proximal$ (MPP) fue introducido por Martinet [6] en 1970 para problemas de optimización convexa y posteriormente fue muy estudiado por Rockafellar [7] para encontrar ceros de operadores monótonos maximales. 
El (MPP) genera una sucesión $\left\{x^{k}\right\}$ dado por $x^{0} \in R^{n} \quad$ ( un punto arbitrario) y

$$
x^{k}=\arg \min \left\{f(x)+\frac{\lambda_{k}}{2}\left\|x-x^{k-1}\right\|^{2}: x \in R^{n}\right\},
$$

donde $\lambda_{k}$ es un parámetro positivo.

Para el caso en que $t$ es convexa se demuestra que la sucesión $\left\{f\left(x^{k}\right)\right\}$ converge al ínfimo de $f \mathrm{y}$ además si el conjunto de soluciones óptimas es no vacío, entonces $\left\{x^{k}\right\}$ converge a una solución del problema, ver Guler [4].

Para el caso cuando $t$ es cuasi-convexa y no diferenciable introducimos el siguiente método:

\section{Método de Optimización Proximal Cuasi-convexo}

Dado una sucesión de parámetros positivos $\left\{\lambda_{k}\right\}$ y un punto inicial

$$
x^{0} \in \mathfrak{R}^{n} .
$$

Criterio de Parada: Para cada $k=1,2, \ldots . .$, si $0 \in \hat{\partial} f\left(x^{k-1}\right)$, entonces finalizar. Caso contrario,

Paso Iterativo: encontrar un $x^{k} \in R^{n}$ tal que

$$
0 \in \hat{\partial}\left(f(.)+\left(\frac{\lambda_{k}}{2}\right)\left\|\cdot-x^{k-1}\right\|^{2}\right)\left(x^{k}\right)
$$

Hacer $k=k+1$ y volver al criterio de parada.

Para lectores interesados en las demostraciones de los resultados de esta sección ver [8].

\subsection{Resultados de convergencia de Fejér}

Definición 4.1.1 Una sucesión $\left\{y^{k}\right\} \subset R^{n}$, es llamada Fejér convergente a un conjunto $U \subseteq R^{n}$, con respecto a la norma euclidiana si :

$\left\|y^{k+1}-u\right\| \leq\left\|y^{k}-u\right\| ; \quad \forall u \in U, \forall k \geq 0$

Teorema 4.1.1 Si la sucesión $\left\{y^{k}\right\}$ es Fejér convergente en un conjunto $U \neq \phi$, entonces $\left\{y^{k}\right\}$ es acotada. Si un punto de acumulación $\bar{y}$ de $\left\{y^{k}\right\}$ pertenece a $U$, entonces $\lim _{k \rightarrow+\infty} y^{k}=\bar{y}$.
Observación 4.1.1 De (4.2) y la diferenciabilidad de $\left(\lambda_{k} / 2\right)\left\|.-x^{k-1}\right\|^{2}$, se tiene que;

$$
0 \in \hat{\partial} f\left(x^{k}\right)+\lambda_{k}\left(x^{k}-x^{k-1}\right),
$$

así, existe $g^{k} \in \hat{\partial} f\left(x^{k}\right)$ tal que

$$
g^{k}=\lambda_{k}\left(x^{k-1}-x^{k}\right) .
$$

\subsection{Resultados de convergencia}

Teorema 4.2.1 Si $\quad f: R^{n} \rightarrow R \cup\{+\infty\}$ es propia, de Lipchitz, acotada inferiormente y semicontinua inferior sobre en $R^{n}$, entonces la sucesión $\left\{x^{k}\right\}$ dado por (4.1) y (4.2) existe.

- Hipótesis A: $f: R^{n} \rightarrow R \cup\{+\infty\}$ es una función acotada inferiormente.

- Hipótesis B: $f: R^{n} \rightarrow R \cup\{+\infty\}$ es localmente lipschitziana y cuasi-convexa.

Como nos interesa la convergencia asintótica del método, asumimos también que en cada iteración $0 \notin \hat{\partial} f\left(x^{k}\right)$, lo cual implica que $x^{k} \neq x^{k-1}, \forall k$.

Proposición 4.2.1 Bajo las hipótesis A y B tenemos que $\left\{f\left(x^{k}\right)\right\}$ es decreciente y convergente.

Definimos el siguiente conjunto:

$$
U=\left\{x \in R^{n} / f(x)<\inf _{j \geq 0} f\left(x^{j}\right)\right\}
$$

Observemos que este conjunto depende del punto inicial $x^{0}$ y la sucesión $\left\{\lambda_{k}\right\}$.

Si $U=\phi$ entonces es posible probar que:
i. $\lim _{k \rightarrow+\infty} f\left(x^{k}\right)=\inf _{x \in R^{n}} f(x)$.
ii. $\left\{x^{k}\right\}$. es limitado.

Se asumirá que $U \neq \phi$ y definimos el siguiente conjunto

$$
V_{k}=\left\{x \in R^{n} / f(x) \leq f\left(x^{k}\right)\right\}
$$

Observamos que $U \subset V_{k}$. De las hipótesis A y $\mathrm{B}$, el conjunto $U$ y $V_{k}$ son no vacíos, cerrados y convexos. 
Teorema 4.2.2 Bajo las hipótesis A y B, la sucesión $\left\{x^{k}\right\}$. generada por el método proximal es Fejér convergente a $\bar{U}$.

Imponemos algunas condiciones a $\left\{\lambda_{k}\right\}$.

Teorema 4.2.3 Suponemos que A y B son satisfechos. Si $0<\lambda_{k}<\bar{\lambda}$, donde $\bar{\lambda}$ es un número real positivo, entonces la sucesión $\left\{x^{k}\right\}$. converge a un punto de $\bar{U}$ y

$$
\lim _{k \rightarrow+\infty} g^{k}=0
$$

para algún $g^{k} \in \hat{\partial} f\left(x^{k}\right)$. Además, $\left\{x^{k}\right\}$ converge a un punto crítico de $t$, esto es, un punto $\bar{x} \in R^{n}$ tal que $0 \in \hat{\partial} f(\bar{x})$.

\section{Implementaciones y Aplicaciones}

En esta sección presentamos algunos experimentos computacionales para resolver problemas irrestrictos con $f$ cuasi-convexa.

Para ello utilizamos el software "Matlab 5.3". Cada problema será ingresado y finalmente presentamos un cuadro con el número de iteraciones y la solución aproximada.

Ejemplo 5.1 Sea $f: R^{n} \rightarrow R$

donde:

$$
f(x, y)=-e^{-x^{2}-y^{2}}
$$

\section{Resultados}

a) Para $\lambda_{k}=1$

$\begin{aligned} & \text { Criterio de error satisfecho Norma gradiente } \\ & =: 0.000415\end{aligned}$
el punto aproximado es: 0.000147
el punto aproximado es: 0.000147
número de iteraciones es: 5
resolvimos el problema aproximadamente

b) Para $\lambda_{k}=1 / 2$

\begin{tabular}{|l|l|}
$\begin{array}{l}\text { Criterio de error satisfecho Normagradiente } \\
=: 0.000906\end{array}$ \\
\hline el punto aproximado es: 0.000320 \\
el punto aproximado es: 0.000320 \\
\hline número de iteraciones es: 5 \\
resolvimos el problema aproximadamente \\
\hline
\end{tabular}

Ejemplo 5.2 Sea $f: R^{n} \rightarrow R$

donde:

\section{Resultados}

$$
f(x)=\left\{\begin{array}{cc}
1-x & ; \quad x<1 \\
x^{2} & ; \quad x \geq 1
\end{array}\right.
$$

a) Para $\lambda_{k}=\frac{1}{k}$

Criterio de error satisfecho Normagradiente $=: 0.0000$

el punto aproximado es: 1.000000

el punto aproximado es: 1.000000

número de iteraciones es: 4

resolvimos el problema aproximadamente

b) Para $\lambda_{k}=1$

Criterio de error satisfecho Normagradiente $=: 0.0000$

el punto aproximado es: 1.000000

el punto aproximado es: 1.000000

número de iteraciones es: 4

resolvimos el problema aproximadamente

c) Para $\lambda_{k}=1 / 2$

Criterio de error satisfecho Normagradiente $=: 0.0000$

el punto aproximado es: 1.000000

el punto aproximado es: 1.000000

número de iteraciones es: 3

resolvimos el problema aproximadamente

\section{APORTE}

El aporte del presente trabajo es de carácter teórico pero con una gran motivación a las aplicaciones. Los resultados de convergencia obtenidos por el método presentado, ver Seción 4, son novedosos y no han sido publicados aún por ninguna revista nacional e internacional. 


\section{CONCLUSIONES}

1. En este trabajo hemos recopilado resultados básicos del subdiferencial de Clarke y la convergencia del método del punto proximal para

adaptarlo a resolver problemas de optimización con funciones objetivo cuasi-convexas.

2. Para una aplicación concreta en problemas de localización y su respectiva implementación computacional y diseño de Software aún está faltando estudiar el método apropiado para resolver los subproblemas (4.2) como también de una caracterización práctica del subdiferencial de Clarke. Esperamos que en futuros trabajos se puedan obtener estos resultados.

3. Otro futuro trabajo sería el estudio de la convergencia del método del Punto Proximal utilizando el subdiferencial de Clarke para resolver problemas de optimización con funciones objetivo y restricciones cuasi-convexas.

\section{REFERENCIAS}

\begin{tabular}{|l|l|}
\hline [1] & $\begin{array}{l}\text { K.J Arrow., and A .C., Enthoven, Quasi- } \\
\text { concave Programming. Econometria. V. 29, } \\
\text { pp. 779-800, 1961. }\end{array}$ \\
\hline [2] & $\begin{array}{l}\text { F.H Clarke, Generalized Gradients and } \\
\text { Application. Transaction of the American }\end{array}$ \\
\hline
\end{tabular}

\begin{tabular}{|l|l|}
\hline & $\begin{array}{l}\text { Mathematical } \\
\text { Society, V.205, pp. 247-262, 1975. }\end{array}$ \\
\hline$[3]$ & $\begin{array}{l}\text { F.H. Clarke, Optimization and Nonsmooth } \\
\text { Analysis (New york:Wiley),1990. }\end{array}$ \\
\hline [4] & $\begin{array}{l}\text { O. Guler On the Convergence of the } \\
\text { Proximal Point Algorithm for Convex } \\
\text { Minimization. SIAM J. Control and } \\
\text { Optimization 29, 403-419, 1991. }\end{array}$ \\
\hline [5] & $\begin{array}{l}\text { Y. Kannai, Concavifiability and Constructions } \\
\text { of Concave Utility Functions. Journal of } \\
\text { Mathematical Economics, V. 4, pp. 1-56, } \\
\text { 1977. }\end{array}$ \\
\hline [6] & $\begin{array}{l}\text { B. Martinet, Régularization d'inequation } \\
\text { Variationelles par Approximations } \\
\text { Successives, R.A.I.R.O., Rech. Oper., 4 } \\
\text { (R3), pp:154-158. 1970 }\end{array}$ \\
\hline [7] & $\begin{array}{l}\text { R. T. Rockafellar, Augmented Lagrangians } \\
\text { and applications of the proximal point } \\
\text { algorithm in convex programming , Math. } \\
\text { Oper. Ress., 1(2), 97-116. 1976. }\end{array}$ \\
\hline [8] & $\begin{array}{l}\text { Tesis de Licenciatura de la FCNM-UNAC: } \\
\text { Tesis: Método del Punto Proximal usando el } \\
\text { Subdiferencial de Clarke. } \\
\text { Autor: Miguel Angel Cano Lengua } \\
\text { Asesor: Dr. Erik Alex Papa Quiroz } \\
\text { Año: Junio del 2011. }\end{array}$ \\
\hline
\end{tabular}

